\title{
Yb-doped glass microcavity laser operation in water
}

\author{
Eric P. Ostby and Kerry J. Vahala* \\ Department of Applied Physics, California Institute of Technology, Pasadena, California 91125, USA \\ *Corresponding author: vahala@caltech.edu
}

Received January 12, 2009; revised February 28, 2009; accepted March 1, 2009; posted March 6, 2009 (Doc. ID 106263); published April 3, 2009

\begin{abstract}
A ytterbium-doped silica microcavity laser demonstrates stable laser emission while completely submerged in water. To our knowledge, it is the first solid-state laser whose cavity mode interacts with water. The device generates more than $2 \mu \mathrm{W}$ of output power. The laser performance is presented, and low-concentration biosensing is discussed as a potential application. (C) 2009 Optical Society of America

OCIS codes: $140.3945,140.3615,160.6060$.
\end{abstract}

Whispering-gallery mode (WGM) microcavities feature an evanescent field component that extends into the surrounding medium [1]. Besides enabling a convenient way to couple optical power, the evanescent field provides a natural means by which resonant modes can interact with their environment. This latter feature is the basis of sensing techniques using passive whispering-gallery microcavities [2,3]. For example, heavy water $\left(\mathrm{D}_{2} \mathrm{O}\right)$ has been detected in normal water through measurement of the quality factor $(Q)$, and interleukin-2 molecules have been detected by measuring a resonance shift $[4,5]$. Theoretical studies have shown that an active microcavity can offer higher sensitivity [6]. As an example of one such device, Lu et al. recently demonstrated a biosensor utilizing the evanescent field component of a dyedoped distributed-feedback laser [7]. In this Letter, a $\mathrm{Yb}: \mathrm{SiO}_{2}$ microcavity laser that operates in water is demonstrated. The work builds upon a recently reported, ytterbium-doped silica microtoroid laser with record-low threshold operation in air [8]. To the best of our knowledge, laser action of a microcavity has not yet been demonstrated in water and provides an important new feature for active biosensing applications.

The microcavity used in this Letter is a silica toroid suspended on a silicon pillar and substrate [9]. These devices feature exceptionally high passive $Q$ factors, making them well suited for laser applications when properly doped. In previous work, the solgel method has been used to dope the microcavity with erbium or ytterbium $[8,10]$. Ytterbium is a more suitable rareearth dopant than erbium for laser operation in water because the absorption coefficient of water is $0.16 \mathrm{~cm}^{-1}$ at $1.04 \mu \mathrm{m}\left(\mathrm{Yb}^{3+}\right.$ emission) compared to $10 \mathrm{~cm}^{-1}$ at $1.55 \mu \mathrm{m}$ ( $\mathrm{Er}^{3+}$ emission) [11]. Furthermore, ytterbium can provide higher gain than erbium, enabling operation at lower microcavity $Q$ values.

The ytterbium-doped silica gain medium is fabricated on a silicon chip according to the solgel chemical synthesis method previously reported [8]. To briefly summarize, tetraethoxysilane, water, isopropyl alcohol, hydrochloric acid, and ytterbium nitrate are mixed in specific quantities at $70^{\circ} \mathrm{C}$ for $3 \mathrm{~h}$ to produce the solgel. The ytterbium concentration for the laser reported in this Letter is $1.1 \times 10^{19} \mathrm{~cm}^{-3}$. Next, the ytterbium-doped solgel is deposited in three layers onto a silicon substrate by spin coating. After each layer is deposited, the thin film is annealed at $1000{ }^{\circ} \mathrm{C}$ in normal atmosphere for $3 \mathrm{~h}$. The refractive index of the $\mathrm{Yb}: \mathrm{SiO}_{2}$ film is 1.46 , according to a spectroscopic ellipsometer measurement (Sentech SE850). Standard photolithography and a buffered oxide wet etch are used to create an array of glass disks with $180 \mu \mathrm{m}$ diameter. These disks are optically isolated from the silicon substrate by a $\mathrm{XeF}_{2}$ dry etch, leaving $\mathrm{Yb}^{3+}: \mathrm{SiO}_{2}$ disks supported by silicon pillars. Finally, a $\mathrm{CO}_{2}$ laser symmetrically reflows each silica microdisk into a smooth microtoroid with $120 \mu \mathrm{m}$ principal diameter. This diameter is optimized for low resonator loss and pump threshold. The reduced index contrast of the toroid resonator in water (0.13) compared to air (0.46) increases both the minimum diameter required to avoid significant radiation loss and also the fraction of power in the evanescent field [12]. A full vectorial finite-element simulation of the fundamental mode shows that the fraction of intensity located outside of the microtoroid increases from $0.6 \%$ to $3.6 \%$ when the toroid is immersed in water.

The $\mathrm{Yb}: \mathrm{SiO}_{2}$ microtoroid laser was characterized first in air and subsequently in water. A single submicrometer diameter fiber taper (Fibercore SMF9805.8-12.5), phase matched to the microcavity's whispering-gallery spatial mode, simultaneously injects pump light and retrieves lasing output as shown in Fig. 1 [13,14]. Finite-element method modeling demonstrates that the toroid mode index increases slightly in water compared to air, because the mode's evanescent component increases and experiences the higher refractive index of water. We are, however, still able to achieve a full range of coupling conditions in water from under- to overcoupled and note that the coupling gap increases in water for a given coupling condition. The taper coupling to the microtoroid is controlled by a three-axis piezo nanopositioning system. A tunable, single-frequency, narrow linewidth $(<300 \mathrm{kHz})$ semiconductor diode laser provides pump excitation in the $970 \mathrm{~nm}$ absorption band of ytterbium. At the fiber output, $\mathrm{Yb}^{3+}: \mathrm{SiO}_{2}$ laser emission at $1040 \mathrm{~nm}$ is separated from unabsorbed 


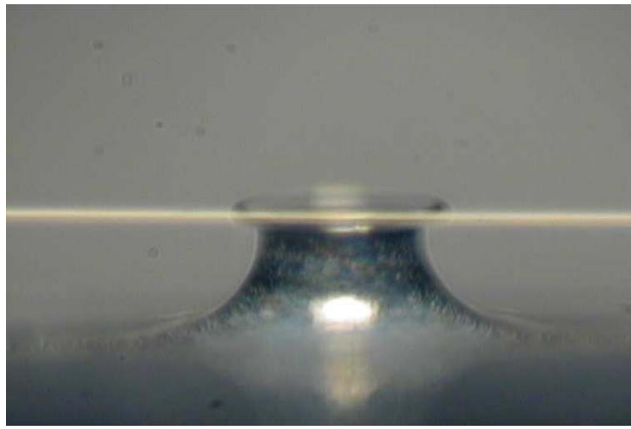

Fig. 1. (Color online) Image of testing setup showing evanescent coupling of fiber taper to $\mathrm{Yb}^{3+}: \mathrm{SiO}_{2}$ microcavity (side view).

pump light by a fiber-based WDM filter ( $45 \mathrm{~dB}$ isolation). For testing in water, a glass coverslip is placed several millimeters above the silicon chip containing the toroid, and the air gap is filled with pure water. Laser output in water was observed in more than 10 microtoroids.

The quality factor of the microcavity pump resonance was measured to ascertain both the doping level as well as the impact of water absorption on the total microcavity $Q$. In air, the $Q$ was measured to be $2.8 \times 10^{5}(\lambda=970.6 \mathrm{~nm})$. This $Q$ factor is dominated by absorption of the $\mathrm{Yb}^{3+}$ ions. $Q$ factors for silica solgel microtoroids have been recorded as high as 2.5 $\times 10^{7}$ [8]. To compare, thermal silica microtoroids routinely have $Q$ factors greater than $1 \times 10^{8}$ [9]. Assuming the measured $Q$ factor $\left(2.8 \times 10^{5}\right)$ is dominated by pump absorption, the doping level is estimated to be $1.1 \times 10^{19} \mathrm{~cm}^{-3}$, in good agreement with the value expected from fabrication $\left(1.2 \times 10^{19} \mathrm{~cm}^{-3}\right)$. In water, the $Q$ factor in the pump band is measured to be $1.3 \times 10^{5}(\lambda=970.8 \mathrm{~nm})$. Figure 2 shows the linewidth measurement of the pump resonance in water in the highly undercoupled regime, which gives the quality factor $(Q=\lambda / \Delta \lambda)$. According to published values of the absorption coefficient of water at $970 \mathrm{~nm}$ $\left(\alpha=45 \mathrm{~m}^{-1}\right)$, the WGM loss in water should cause a reduction in measured $Q$ to only $2.3 \times 10^{5}$ [11]. Therefore, there exists an additional source of absorption, such as external contaminants carried to the micro-

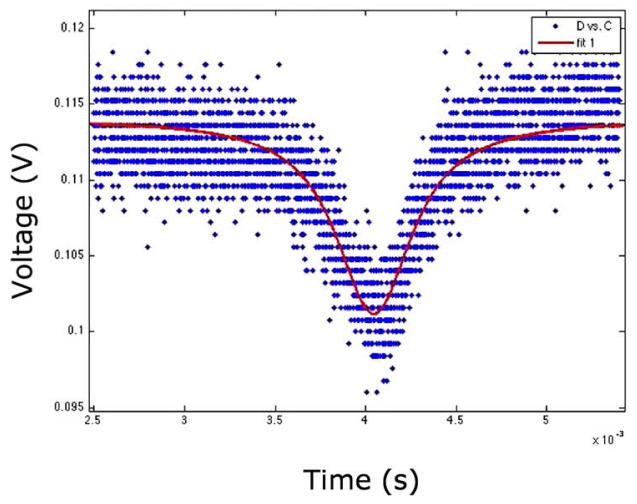

Fig. 2. (Color online) Plot of microtoroid pump-mode resonance in water with Lorenzian fit for the undercoupled condition $\left(Q=1.3 \times 10^{5}\right.$ at $\left.\lambda=970.8 \mathrm{~nm}\right)$. Data are taken by measuring the transmitted power along the fiber taper while the pump laser wavelength is scanned in time.

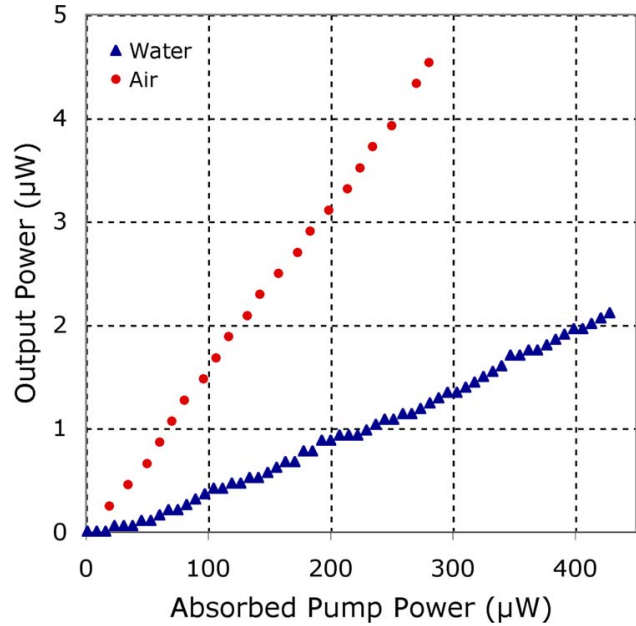

Fig. 3. (Color online) Comparison of measured laser output power as a function of absorbed pump power for $120 \mu \mathrm{m}$ diameter $\mathrm{Yb}^{3+}: \mathrm{SiO}_{2}$ microtoroid in air and water.

toroid by water. This is currently under investigation.

The $\mathrm{Yb}: \mathrm{SiO}_{2}$ microtoroid laser's output power was measured as a function of the absorbed pump power for operation in air and water (see Fig. 3). The microtoroid cavity supports both clockwise and counterclockwise modes, but we only record the power of the clockwise mode. The laser turn-on pump threshold is $3 \mu \mathrm{W}$ in air and $14 \mu \mathrm{W}$ in water, with corresponding slope efficiencies of $1.6 \%$ (air) and $0.5 \%$ (water). In water, there is a clear threshold for laser operation as well as a linear dependence of output power on absorbed pump power (see Fig. 4). The maximum output power in water is $2 \mu \mathrm{W}$. Table 1 summarizes the laser performance in air and water. Following the work of Min et al., the predictions of a laser rate equation model and experimental results are compared with reasonable agreement [15]. In water (air), the experimental threshold, $14 \mu \mathrm{W}(3 \mu \mathrm{W})$, is in reasonable agreement with the prediction of the model, $4 \mu \mathrm{W}(1 \mu \mathrm{W})$.

In conclusion, we have demonstrated a solid-state microcavity laser whose cavity mode extends into wa-

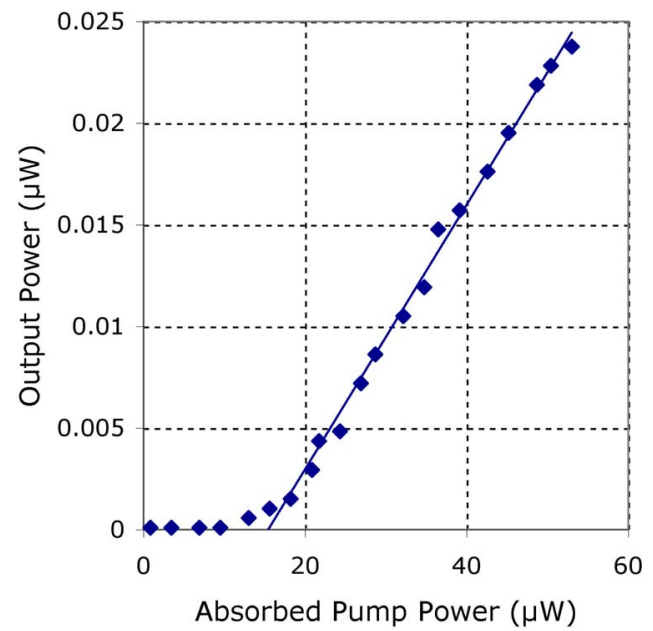

Fig. 4. (Color online) Closeup of the laser threshold in water shown in Fig. 3. 
Table 1. Comparison of Microtoriod Laser Results in Air and Water

\begin{tabular}{|c|c|c|c|c|}
\hline Environment & $\begin{array}{l}\text { Quality } \\
\text { Factor }\end{array}$ & $\begin{array}{c}\text { Pump } \\
\text { Threshold } \\
(\mu \mathrm{W})\end{array}$ & $\begin{array}{c}\text { Slope } \\
\text { Efficiency } \\
(\%)\end{array}$ & $\begin{array}{c}\text { Evanescent } \\
\text { Fraction } \\
\text { of WGM } \\
(\%)\end{array}$ \\
\hline Air & $2.8 \times 10^{5}$ & 3 & 1.6 & 0.6 \\
\hline Water & $1.3 \times 10^{5}$ & 14 & 0.5 & 3.6 \\
\hline
\end{tabular}

ter. The laser generates single-frequency light with an output power of $2 \mu \mathrm{W}$ and a threshold of $14 \mu \mathrm{W}$. The Schawlow-Townes equation, $\Delta \nu=\pi h \nu^{3} / P Q^{2}$, quantifies the fundamental laser linewidth for a single-mode laser ignoring any technical noise [16]. Given the experimental values for laser power $(2 \mu \mathrm{W})$ and the water absorption limited quality factor $\left(6.5 \times 10^{5}\right)$ at $1.04 \mu \mathrm{m}$, we estimate that the fundamental linewidth of the ytterbium silica laser presented in this Letter is $60 \mathrm{kHz}$, which is significantly narrower than the linewidth of an ultra-high- $Q$ passive microcavity (of order $1 \mathrm{MHz}$ ). As a result, this laser can have higher sensitivity to biological molecules in aqueous solution than a passive resonator. The laser threshold, output power, efficiency, and emission frequency can be modified by a molecule located close to the microtoroid, providing several possible sensing mechanisms. Further ways to improve the $Q$ factor and output power, both of which decrease the laser linewidth, are under study.

The authors thank A. Armani (University of Southern California) for advice on microtoroid testing in water. This work was financially supported by the Defence Advanced Research Projects Agency (DARPA) Center for Optofluidic Integration.

\section{References}

1. K. J. Vahala, Nature 424, 839 (2003).

2. S. Arnold, M. Khoshsima, I. Teraoka, S. Holler, and F. Vollmer, Opt. Lett. 28, 272 (2003).

3. K. De Vos, I. Bartolozzi, E. Schacht, P. Bienstman, and R. Baets, Opt. Express 15, 7610 (2007).

4. A. M. Armani and K. J. Vahala, Opt. Lett. 31, 1896 (2006).

5. A. M. Armani, R. P. Kulkarni, S. E. Fraser, R. C. Flagan, and K. J. Vahala, Science 317, 783 (2007).

6. J. Yang and L. J. Guo, IEEE J. Sel. Top. Quantum Electron. 12, 143 (2006).

7. M. Lu, S. Choi, C. J. Wagner, J. G. Eden, and B. T. Cunningham, Appl. Phys. Lett. 92, 261502 (2008).

8. E. P. Ostby, L. Yang, and K. J. Vahala, Opt. Lett. 32, 2650 (2007)

9. D. K. Armani, T. J. Kippenberg, S. M. Spillane, and K. J. Vahala, Nature 421, 925 (2003).

10. L. Yang, T. Carmon, B. Min, S. M. Spillane, and K. J. Vahala, Appl. Phys. Lett. 86, 091114 (2005).

11. G. M. Hale and M. R. Querry, Appl. Opt. 12, 555 (1973).

12. A. M. Armani, D. K. Armani, B. Min, K. J. Vahala, and S. M. Spillane, Appl. Phys. Lett. 87, 151118 (2005).

13. S. M. Spillane, T. J. Kippenberg, O. J. Painter, and K. J. Vahala, Phys. Rev. Lett. 91, 043902 (2003).

14. M. Cai, O. Painter, and K. J. Vahala, Phys. Rev. Lett. 85, $74(2000)$.

15. B. K. Min, T. J. Kippenberg, L. Yang, K. J. Vahala, J. Kalkman, and A. Polman, Phys. Rev. A 70, 033803 (2004).

16. A. L. Schawlow and C. H. Townes, Phys. Rev. 112, 1940 (1958). 